COMMUNICATIONS IN

ANALYSIS AND GEOMETRY

Volume 13, Number 4, 671-695, 2005

\title{
A Connectedness principle in the geometry of positive curvature
}

\author{
Fuquan Fang, ${ }^{1}$ Sérgio Mendonça, Xiaochun Rong ${ }^{2}$
}

The main purpose of this paper is to develop a connectedness principle in the geometry of positive curvature. In the form, this is a surprising analog of the classical connectedness principle in complex algebraic geometry. The connectedness principle, when applied to totally geodesic immersions, provides not only a uniform formulation for the classical Synge theorem, the Frankel theorem and a recent theorem of Wilking for totally geodesic submanifolds, but also new connectedness theorems for totally geodesic immersions in the geometry of positive curvature. However, the connectedness principle may apply in certain cases which do not require the existence of totally geodesic immersions.

\section{Introduction.}

In algebraic geometry, the connectedness principle is a theme that may be stated as follows (cf. [14]): Given a suitably "positive" embedding of manifold of codimension $d, D \hookrightarrow P$, and a proper morphism, $f: N^{n} \rightarrow P$,

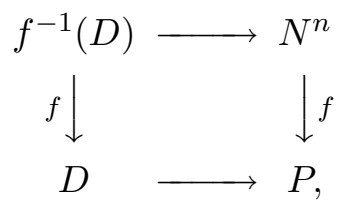

the homotopy groups should satisfy that $\pi_{i}\left(N^{n}, f^{-1}(D)\right) \cong \pi_{i}(P, D)$ for $i \leq n-d-$ "defect", where the defect can be measured by the lack of positivity of $N$ in $P$, the singularities of $N$ and the dimension of the fibers of $f$. The most basic case is when $P=\mathbb{P}^{m} \times \mathbb{P}^{m}$, the product of the projective space, $D=\Delta$, the diagonal submanifold $P$, and $N$ is the product

\footnotetext{
${ }^{1}$ Supported by CNPq of Brazil, NSFC Grant 19741002, RFDP and Qiu-Shi Foundation of China

${ }^{2}$ Supported partially by NSF Grant DMS 0203164 and a research found from Capital normal university
} 
of subvarieties. In this case, the connectedness principle easily yields several classical connectedness theorems; see [12] for details.

In this paper, we develop a surprisingly "similar" connectedness principle in the geometry of positive (sectional or $k$-th Ricci) curvature (cf. [34, 31]), where $\mathbb{P}^{m}$ is replaced by a closed manifold of positive curvature, and the "subvarieties" and "morphism" are replaced by an embedded, or a minimal immersed closed submanifold with large asymptotic index (see below). Note that all manifolds considered in the paper are connected. The asymptotic index of an immersion $f: N \rightarrow M$ is defined by $\nu_{f}=\min _{x \in N} \nu_{f}(x)$, where $\nu_{f}(x)$ is the maximal dimension of a subspace of $T_{x} N$ on which the second fundamental form vanishes (cf. p 188 of [8]). Clearly, $f$ is a totally geodesic immersion if and only if $\nu_{f}=\operatorname{dim}(N)$. However, an isometric immersion with non-degenerate second fundamental form may satisfy that $\nu_{f}>0$ (e.g., any minimal immersion satisfies that $\nu_{f} \geq 1$, and any isoparametric minimal hypersurface with two distinct principle curvature has a non-degenerate second fundamental form and $\left.\nu_{f} \geq \frac{1}{2} \operatorname{dim}(N)\right)$.

The connectedness principle of positive curvature, when applied to totally geodesic immersions, provides with not only a uniform formulation for the classical Synge theorem, the Frankel theorem and a recent theorem of Wilking for totally geodesic submanifolds, but also new connectedness theorems for totally geodesic immersions.

Substantial applications of the connectedness principle have been obtained in the classifications for the positively curved manifolds with large isometry groups (cf. [6, 7, 33]). In all these works, the existence of 'a totally geodesic submanifold of small codimension' is being proved and plays a crucial role.

However, the connectedness principle may apply in a more general situation which may not guarantee the existence of a totally geodesic immersion. This is important to note because the requirement of 'a totally geodesic submanifold of small codimension' may be quite restrictive. The following is a typical example of such an application (see Theorems 0.1-0.3).

A map from $N$ to $M$ is called $(i+1)$-connected if it induces an isomorphism up to the $i$-th homotopy group and a surjective homomorphism on the $(i+1)$-th homotopy group.

The following result is a consequence of Theorems 0.5 and 0.8 (see below).

Theorem 0.1 (Connectedness - Isometric immersion). Let $M^{m}$ be a closed simply connected manifold of positive sectional curvature. If $f: N^{n} \rightarrow M^{m}$ is an isometric immersion of a closed manifold with asymptotic index $\nu_{f}$, then $f$ is $\left(2 \nu_{f}-m+1\right)$-connected. 
Note that Theorem 0.1 is false if one removes the restriction on simply connectedness (e.g. take $N$ to be the universal Riemannian covering of $M$ ); see Theorem 4.1 for the non-simply connected case.

For an isometric immersion, $f: N \rightarrow M$, the asymptotic index may be estimated in terms of the extrinsic curvature of $f([5,8])$. For any two plane $\sigma$ in $T_{x}(N), x \in N$, the extrinsic curvature of $f$ is defined by

$$
\operatorname{excur}_{f}(\sigma)=\sec _{N}(\sigma)-\sec _{M}\left(f_{*}(\sigma)\right)=\langle\alpha(X, X), \alpha(Y, Y)\rangle-\|\alpha(X, Y)\|^{2},
$$

where $\alpha$ is the second fundamental form and $\{X, Y\}$ denotes any orthonormal basis for $\sigma$. If $\operatorname{excur}_{f}(\sigma) \leq 0$ for all $\sigma$, then we say that $f$ has non-positive extrinsic curvature. A non-trivial simple example is the unit normal bundle of the Veronesse surface in the unit sphere $S^{4}$. Obviously, a totally geodesic immersion has zero extrinsic curvature, but the converse may not be true.

By [8] (see Theorems 4.2), from Theorem 0.1, we conclude:

Theorem 0.2 (Connectedness - Non-positive extrinsic curvature). Let $M^{n+p}$ be a closed simply connected manifold of positive sectional curvature, and let $f: N^{n} \rightarrow M^{n+p}$ be an isometric immersion. If $f$ has non-positive extrinsic curvature, then $f$ is $(n-3 p+1)$-connected.

Note that Theorem 0.2 asserts the connectedness property from an assumption solely on the sectional curvature. Moreover, Theorem 0.2 is false if one replaces "positive" curvature by "non-negative curvature" (e.g. $f$ is the inclusion of $S^{n}$ into $S^{n} \times S^{2}$ for $n \geq 7$ ).

An interesting consequence of Theorem 0.2 is:

Theorem 0.3 (Rigidity - Non-positive extrinsic curvature). Let $M^{n+p}$ be a closed simply connected manifold of positive sectional curvature, and let $N^{n} \rightarrow M^{n+p}$ be an isometric immersion with non-positive extrinsic curvature. If $n \geq 7 p-2$, then $N^{n}$ is homeomorphic to a sphere if and only if $M^{n+p}$ is homeomorphic to a sphere.

Perhaps the first evidence for the analog of the connectedness principle in the geometry of positive curvature goes back to a Synge type theorem ([9]) on the intersection of closed totally geodesic submanifolds; whose counterpart in algebraic geometry is the Bézout Theorem (see [12, page 17]). The latest evidence, that really caught our attention, is a theorem of Wilking ([33], comparable to Theorem 0.8), which may be considered as a counterpart of the Barth-Lefschetz type theorem (cf. [29]).

We now begin to state the connectedness principle of positive curvature. 
Theorem A. (Connectedness principle of positive curvature) Let $M^{m}$ be a closed manifold of positive sectional curvature, and let $\Delta$ denote the diagonal submanifold of $M^{m} \times M^{m}$. Let $f: N^{n} \rightarrow M^{m} \times M^{m}$ be an isometric immersion of a closed manifold with asymptotic index $\nu_{f}$. Then, the following statements hold:

(A1) If $\nu_{f}>m$, then $f^{-1}(\Delta)$ is non-empty.

(A2) If $\nu_{f}>m+1$ and $M^{m}$ is simply connected, then $f^{-1}(\Delta)$ is connected.

If $f$ is also a minimal immersion, then

(A3) For $\nu_{f}>m+i$, there is an exact sequence of homotopy groups $\pi_{i}\left(f^{-1}(\Delta)\right) \rightarrow \pi_{i}\left(N^{n}\right) \stackrel{\left(p_{1} f\right)_{*}-\left(p_{2} f\right)_{*}}{\longrightarrow} \pi_{i}\left(M^{m}\right) \rightarrow \pi_{i-1}\left(f^{-1}(\Delta)\right) \rightarrow \cdots$

where $p_{1}, p_{2}$ are the projections of $M^{m} \times M^{m}$ to the first and second factors respectively.

Note that the strict inequality condition above may not be improved to the weak inequality if $f$ is a totally geodesic immersion. For instance, if $M^{2 m}$ admits a free isometric involution $\phi$, then $f: M^{2 m} \rightarrow M^{2 m} \times M^{2 m}$ defined by $f(x)=(x, \phi(x))$ is totally geodesic and satisfies $f^{-1}(\Delta)=\emptyset$; compare to (A1).

However, below, in the most interesting cases, Theorem A is sharpened with all strict inequalities replaced by weak inequalities (see Theorem B). Moreover, there is an additional property (see B4).

For a map $f: Y \rightarrow X$, let $X_{f}=X \cup_{f}(Y \times[0,1])$ denote the mapping cylinder of $f$. We will use $\pi_{i}(X, Y)$ to denote the relative homotopy group $\pi_{i}\left(X_{f}, Y\right)$, called the $i$-th homotopy group of $f$.

Theorem B. (Connectedness principle of positive curvaturesubmanifolds) Let the assumptions be as in Theorem A. If, in addition, $N^{n}=N_{1} \times N_{2}$ and $f=\left(f_{1}, f_{2}\right)$ with asymptotic index $\nu_{f}$, then

(B1) If $\nu_{f} \geq m$, then $f^{-1}(\Delta)$ is non-empty.

(B2) If $\nu_{f} \geq m+1$ and $M^{m}$ is simply connected, then $f^{-1}(\Delta)$ is connected. If $f$ is either a minimal immersion or $f=\left(f_{1}, f_{1}\right)$, where $f_{1}$ is an embedding, then

(B3) For $\nu_{f} \geq m+i$, there is an exact sequence

$$
\pi_{i}\left(f^{-1}(\Delta)\right) \rightarrow \pi_{i}\left(N^{n}\right) \stackrel{\left(p_{1} f\right)_{*}-\left(p_{2} f\right)_{*}}{\longrightarrow} \pi_{i}\left(M^{m}\right) \rightarrow \pi_{i-1}\left(f^{-1}(\Delta)\right) \rightarrow \cdots
$$


(B4) There are natural isomorphisms

$$
\pi_{i}\left(N_{1}, f^{-1}(\Delta)\right) \rightarrow \pi_{i}\left(M^{m}, N_{2}\right), \quad \pi_{i}\left(N_{2}, f^{-1}(\Delta)\right) \rightarrow \pi_{i}\left(M^{m}, N_{1}\right)
$$

for $i \leq \nu_{f}-m$ and a surjection for $i=\nu_{f}-m+1$, where $\pi_{i}\left(N_{j}, f^{-1}(\Delta)\right)$ is understood as the $i$-th homotopy group of the composition map $f^{-1}(\Delta) \subset N^{n} \stackrel{p_{j}}{\longrightarrow} N_{j}$.

Some comments on Theorem B are in order.

First, (B1) is a strengthened version of the following Synge type theorem for embedded totally geodesic submanifolds.

Theorem 0.4 (Generalized Frankel Theorem). Let $M^{m}$ be a closed manifold of positive sectional curvature, and let $f_{i}: N_{i} \rightarrow M^{m}(i=1,2)$ be an isometric immersion of a closed submanifold with asymptotic index $\nu_{f_{i}}$. If $\nu_{f_{1}}+\nu_{f_{2}} \geq m$, then $f_{1}\left(N_{1}\right) \cap f_{2}\left(N_{2}\right) \neq \emptyset$.

Secondly, (B2) implies a regularity result for isometric immersions; whose counterpart in algebraic geometry is the Fulton-Hansen regularity theorem $([12])$.

Theorem 0.5 (Regularity). Let $M^{m}$ be a closed simply connected manifold of positive sectional curvature. Let $f: N^{n} \rightarrow M^{m}$ be an isometric immersion of a closed manifold. Then, $f$ must be an embedding, provided that the asymptotic index $\nu_{f}>m / 2$.

Note that the condition ' $\nu_{f}>m / 2$ ' is optimal for $m=2$; on a threeaxial ellipsoids there are many closed geodesics that have self-intersections (cf. [21, 24]). Moreover, Theorem 0.5 does not hold if $M^{m}$ is not simply connected (e.g. the composition of the inclusion with the regular covering $f$ : $S^{3} \rightarrow \mathbb{R} P^{3} \subset \mathbb{R} P^{5}$ is a totally geodesic immersion, but not an embedding.)

Another consequence of (B2) is:

Theorem 0.6. Let $M^{m}$ be a closed simply connected manifold of positive sectional curvature. Let $f_{j}: N_{j} \rightarrow M^{m}$ be a closed isometric immersion with asymptotic index $\nu_{f_{j}}, j=1,2$. If $\nu_{f_{1}}+\nu_{f_{2}} \geq m+1$, then both $f_{1}^{-1}\left(f_{2}\left(N_{2}\right)\right)$ and $f_{2}^{-1}\left(f_{1}\left(N_{1}\right)\right)$ are connected.

Note that the requirement ' $\nu_{f_{1}}+\nu_{f_{2}} \geq m+1$ ' is optimal; there are two totally geodesic embedded spheres $S^{2 n}$ in $S^{4 n}$ of constant curvature with intersection set two points. 
Theorem 0.6 may be compared with a Bertini-type theorem which asserts the same conclusion when one replaces $M$ by $\mathbb{P}^{m}$ and $N$ by an algebraic subvariety $([12])$.

Thirdly, (B3) (resp. (A3)) may be considered as the counterpart of a connectedness theorem of Deligne, a generalization of a theorem of FultonHansen (cf. [18, page 27]).

Fourthly, (B4) may be considered as an analog of a connectedness theorem of Fulton-Lazarsfeld [14] for local complete intersections. An immediate consequence of (B4) is an analog of the Barth-Lefschetz type theorem in algebraic geometry (cf. [2, 3, 22]):

Theorem 0.7. Let $M^{m}$ be a closed manifold of positive sectional curvature, and let $N_{j} \hookrightarrow M^{m}$ be a closed embedded submanifold of asymptotic index $\nu_{j}$ $(j=1,2)$. If either $N_{j}(j=1,2)$ is minimal or $N_{1}=N_{2}$, then the following natural homomorphisms,

$$
\pi_{i}\left(N_{1}, N_{1} \cap N_{2}\right) \rightarrow \pi_{i}\left(M^{m}, N_{2}\right), \quad \pi_{i}\left(N_{2}, N_{1} \cap N_{2}\right) \rightarrow \pi_{i}\left(M^{m}, N_{1}\right),
$$

are isomorphisms for $i \leq \nu_{1}+\nu_{2}-m$ and are surjections for $i=\nu_{1}+\nu_{2}-m+1$.

Theorem 0.7 implies the following generalized Wilking's theorem (the Wilking's theorem asserts the connectedness properties for totally geodesic submanifolds, [33]). As mentioned earlier, the Wilking's theorem may be considered as the counterpart of some Barth-Lefschetz type theorem (cf. [2, 29]).

Theorem 0.8 (Generalized Wilking Theorem). Let $M^{m}$ be a closed manifold of positive sectional curvature, and let $N_{j}$ be a closed embedded submanifold of asymptotic index $\nu_{j}, j=1,2$. Then,

(0.8.1) the inclusion $i_{2}: N_{2} \hookrightarrow M^{m}$ is $\left(2 \nu_{2}-m+1\right)$-connected.

(0.8.2) If $N_{1}$ and $N_{2}$ are both minimal, then $N_{1} \cap N_{2} \hookrightarrow N_{1}$ is $\left(\nu_{1}+\nu_{2}-m\right)$ connected.

As mentioned earlier, Theorem 0.1 follows from Theorems 0.5 and 0.8 .

Another case where (A1) is sharpened is indeed a reformulation of the classical Synge theorem ([32]). This was pointed out to us by Karsten Grove.

Theorem 0.9 (Weinstein). Let $M$ be a closed orientable evendimensional manifold of positive sectional curvature. For any orientation preserving isometry $\phi$, the totally geodesic embedding $f: M \rightarrow M \times M$ by $f(x)=(x, \phi(x))$ satisfies $f^{-1}(\Delta) \neq \emptyset$. In particular, $M$ is simply connected. 
The connectedness principle also extends to closed manifolds of positive $k$-th Ricci curvature conditions (cf. [34, 31]). Here, it is only in the form found in Theorem B. For an $m$-manifold and $1 \leq k \leq m-1$, we say that the $k$ th Ricci curvature is positive if for any orthonormal $(k+1)$ vectors, $v_{1}, \ldots, v_{k+1}$, we have $(R(\cdots)$ is the curvature tensor $)$ :

$$
\sum_{j=1}^{k+1} R\left(v_{i}, v, v, v_{i}\right)>0, \quad \text { for all } \mathrm{v} \text { spanned by } v_{1}, \ldots, v_{k_{+1}} \text {. }
$$

The 1-st Ricci is the sectional curvature and $(m-1)$-th Ricci is the Ricci curvature.

The following connectedness principle can be viewed as a generalization of Theorem B.

Theorem C. (Connectedness principle of positive $k$-th Ricci curvature) Let $M^{m}$ be a closed manifold of positive $k$-th Ricci curvature, and let $f=\left(f_{1}, f_{2}\right): N_{1} \times N_{2} \rightarrow M^{m} \times M^{m}$, where $f_{j}: N_{j} \rightarrow M^{m}$ is an isometric immersion of a closed manifold with asymptotic index $\nu_{f_{j}}, j=1,2$. Then, the following properties hold true $\left(\nu_{f}=\nu_{f_{1}}+\nu_{f_{2}}\right)$ :

(C1) If $\nu_{f} \geq m+k-1$, then $f^{-1}(\Delta)$ is non-empty.

(C2) If $\nu_{f} \geq m+k$ and $M^{m}$ is simply connected, then $f^{-1}(\Delta)$ is connected.

If $f$ is either a minimal immersion or $f=\left(f_{1}, f_{1}\right)$, where $f_{1}$ is an embedding, then

(C3) For $\nu_{f} \geq m+k+i-1$, there is an exact sequence

$$
\pi_{i}\left(f^{-1}(\Delta)\right) \rightarrow \pi_{i}(N) \stackrel{\left(p_{1} f\right)_{*}-\left(p_{2} f\right)_{*}}{\longrightarrow} \pi_{i}\left(M^{m}\right) \rightarrow \pi_{i-1}\left(f^{-1}(\Delta)\right) \rightarrow \cdots
$$

(C4) For $i \leq \nu_{f}-m-k+1$, there are natural isomorphisms $\pi_{i}\left(N_{1}, f^{-1}(\Delta)\right) \rightarrow \pi_{i}\left(M^{m}, N_{2}\right)$ and $\pi_{i}\left(N_{2}, f^{-1}(\Delta)\right) \rightarrow \pi_{i}\left(M^{m}, N_{1}\right)$, and a surjection for $i=\nu-m-k+2$.

Note that from $(\mathrm{C} 1)-(\mathrm{C} 4)$, one may conclude results similar to Theorems $0.3-0.8$ (see comments following Theorem B). Because this may be done in a relatively straightforward manner, we omit it here.

In the case of positive Ricci curvature, following the proof of $(\mathrm{C} 1)$, we obtain

Corollary 0.10. Let $M$ be a closed manifold of positive Ricci curvature. If $N$ is a closed embedded minimal hypersurface, then the inclusion map induces a surjection on the fundamental groups. 
Note that Corollary 0.10 recovers the case of a theorem of Frankel (cf. [10], see [27] for a generalization).

Some remarks on the above results are in order.

Remark 0.11. It may be worthwhile to emphasize that the consideration of a closed isometric immersion with asymptotic index in Theorems A-C (instead of a totally geodesic submanifold) is essential for new applications of (B2) (e.g., Theorems 0.1-0.3 and 0.5). The motivation for an "immersion" is from the uniform formulation of connectedness theorems in algebraic geometry seen at the beginning of this paper ([12, 14]). As a by-product, we obtain strengthened versions of some known connectedness theorems.

Remark 0.12. Theorems A-C may hold under some partially positive curvature conditions (e.g. almost everywhere positive curvature). For instance, Theorems 0.5 and 0.6 hold if $M$ has positive sectional curvature outside $N$ or if $M$ has positive sectional curvature at points in $N$.

Remark 0.13. Theorem B may be optimal in the sense that the weak inequality conditions cannot be improved in most general situations. However, with additional assumptions, the weak inequality conditions may be improved. For instance, in Theorems $0.4-0.8$, if one assumes in addition that $M^{m}$ admits an isometric compact Lie group $G$-action and $N^{n}$ is fixed pointwisely by $G$, then the dimension restriction may be improved (cf. [33]).

Remark 0.14. Let $M=G / H$ be a compact type homogeneous space with normal metric. Let $r(G)$ denote the rank of the compact Lie group $G$. Then, the $k$ th Ricci curvature of $G / H$ is positive for all $k \geq r(G)+1$, showing that Theorem $\mathrm{C}$ may be applied to immersions in homogeneous spaces.

Remark 0.15. The connectedness principle may be extended (with identical proofs) to Finsler manifolds whose flag curvatures are bounded from below uniformly by a positive constant (cf. [1, page 221-223]).

We now give an indication for the proofs of Theorems A-C. First, we apply the Morse theory to the energy function on the twisted path space which is the pullback from the path space $P(M)$ on $M$ via an immersion $f: N \rightarrow M \times M$, and estimate a lower bound for the indices of non-trivial critical points, see Lemma 2.1. Then, we derive the desired connectedness properties from the various lower bounds, see Theorem 1.1.

We would like to mention that in [15], Karsten Grove applied the Morse theory to the energy function on the subspace of $P(M)$ consisting of paths 
whose ends are in a closed submanifold of $M$ and derived, under the assumption that the energy function has no non-trivial critical point, an exact sequence similar to (B3) (compare to Remark 0.11).

This paper was partially inspired by Theorem 0.8 for totally geodesic submanifolds due to Wilking ([33]). Its origin stems from the recent works of $[6,7]$ that used Theorem 0.8 for totally geodesic submanifolds. Since [33] had not yet been available to us, we wrote a proof of Theorem 0.8 for ourselves; that was the starting point of the present paper.

The rest of the paper is organized as follows:

In Section 1, we apply Morse theory to the twisted path space $P(M, f)$.

In Section 2, we estimate the lower index bound for energy function on $P(M, f)$.

In Section 3, we prove Theorems A-C.

In Section 4, we prove Theorems 0.1-0.9.

In Section 5, we provide an application of the connectedness theorems.

\section{Morse Theory on Path Spaces.}

The Morse theory on path space $P(M)$ with various boundary conditions has been well-developed (cf. [25]). In this section, we will apply the Morse theory to the twisted path space i.e. the pullback from $P(M)$ via an isometric immersion in $M \times M$. We use the word 'twisted' to mean that it may not be a subspace of $P(M)$ (compare to [15]).

The main result of this section is Theorem 1.1 which concerns with relations between connectedness properties and various lower index bounds on the non-trivial critical points of the energy function. Theorem 1.1 will be used in the proof of Theorems A, B and C.

\subsection{The path space $P(M, f)$.}

For a connected complete Riemannian manifold $M$, let $P(M)$ denote the space of piecewise smooth paths in $M$ equipped with the metric topology with the metric given by

$d\left(\gamma_{0}, \gamma_{1}\right)=\left(\int_{0}^{1}\left(\left|\dot{\gamma}_{0}(t)\right|-\left|\dot{\gamma}_{1}(t)\right|\right)^{2} d t\right)^{\frac{1}{2}}+\max _{0 \leq t \leq 1} d_{M}\left(\gamma_{0}(t), \gamma_{1}(t)\right), \gamma_{0}, \gamma_{1} \in P(M)$

Note that the integral is well-defined even though $\dot{\gamma}_{i}(i=1,2)$ may not be defined on finitely many points in $[0,1]$. The projection map, $p: P(M) \rightarrow$ 
$M \times M$ by $p(\gamma)=(\gamma(0), \gamma(1))$, defines the Serre fibration, $\Omega(M) \rightarrow P(M) \rightarrow$ $M \times M$, with fiber $\Omega(M)$, the loop space of $M$ with a fixed base point.

Let $N$ be a manifold, and let $f: N \rightarrow M \times M$ be any smooth map. Let $\Omega(M) \rightarrow P(M, f) \rightarrow N$ denote the pullback fibration by $f$. Clearly, $P(M, f) \subset N \times P(M)$ consists of $(x, \gamma)$ such that $f(x)=(\gamma(0), \gamma(1))$ and is equipped with the induced topology.

In the rest of this section, we assume that $M$ and $N$ are closed manifolds.

\subsection{The Morse theory on $P(M, f)$.}

We will study $P(M, f)$ via the Morse theory for the energy function $E(x, \gamma)=\frac{1}{2} \int_{0}^{1}|\dot{\gamma}|^{2} d t$. From the standard first variation formula, one sees that (cf. [15])

(1.1) Any critical point $(x, \gamma)$ of $E$ is a geodesic with $(\dot{\gamma}(0),-\dot{\gamma}(1)) \perp$ $f_{*}\left(T_{x}(N)\right)$.

Theorem 1.1. Let $M$ and $N$ be closed manifolds, and $f: N \rightarrow M \times M$ an isometric immersion. Let $\Delta \subset M \times M$ be the diagonal. Assume that every non-trivial critical point $(x, \gamma)$ (i.e. $\gamma$ is not a point) of $E$ has index $I_{\gamma} \geq \lambda_{0}$. Then

(1.2.1) If $\lambda_{0} \geq 1$, then $f^{-1}(\Delta) \neq \emptyset$.

(1.2.2) If $\lambda_{0} \geq 2$ and $M$ is simply connected, then $f^{-1}(\Delta)$ is connected.

If $f$ is, in addition, either a minimal immersion or $f=f_{1} \times f_{1}: N=$ $N_{1} \times N_{1} \rightarrow M \times M$ where $f_{1}$ is an embedding, then

(1.2.3) $\pi_{i}\left(P(M, f), f^{-1}(\Delta)\right)=0$ for all $i<\lambda_{0}$.

(1.2.4) If $\lambda_{0} \geq i$, then there is an exact sequence of homotopy groups,

$$
\pi_{i}\left(f^{-1}(\Delta)\right) \rightarrow \pi_{i}(N) \stackrel{\left(p_{1} f\right)_{*}-\left(p_{2} f\right)_{*}}{\longrightarrow} \pi_{i}(M) \rightarrow \pi_{i-1}\left(f^{-1}(\Delta)\right) \rightarrow \cdots
$$

where $p_{1}, p_{2}$ are the projections of $M \times M$ to the first and second factors respectively.

Proof.

(1.2.1) If $f^{-1}(\Delta)=E^{-1}(0)=\emptyset$, then $E$ has a positive absolute minimal value at some non-trivial critical point $(x, \gamma)$ and thus its index is zero; a contradiction. 
(1.2.2) We use the standard technique of a finite dimensional approximation by Morse (cf. [25, 29]). Because of this, we may omit some details.

For fixed $c>0$, put $P_{c}(M, f)=E^{-1}([0, c))$, an open subset of $P(M, f)$. Let $B_{c}(M, f) \subset P_{c}(M, f)$ consist of piecewise smooth geodesics with $k$ broken points (each piece with length less than the injectivity radius). Then, $B_{c}(M, f)$ has the same homotopy type as $P_{c}(M, f)$ for $k$ sufficiently large. Note that $B_{c}(M, f)$ may be identified with an open submanifold of product $N \times M \times \cdots \times M$ (with $k$ copies of $M$ ). Moreover, $E$ is a proper function when restricted to $B_{c}(M, f)$, and $\left.E\right|_{B_{c}(M, f)}$ and $\left.E\right|_{P_{c}(M, f)}$ have the same critical points with identical indices (cf. [25, Sec. 14 and 16]).

Suppose that $f^{-1}(\Delta)$ is not connected. By definition, there exist disjoint non-empty compact subsets $A$ and $B$ such that $A \cup B=f^{-1}(\Delta)$. Identify $f^{-1}(\Delta)$ with constant paths in $P(M, f)$. Fix a point $p \in A$ and $q \in B$.

Since $M$ is simply connected, it follows that the loop space $\Omega M$ is path connected. Therefore, $P(M, f)$ is also path connected. Thus, there is a path $\gamma_{0}$ in $P(M, f)$ joining $p$ and $q$. By the above, we may assume a path $\bar{\gamma}_{0} \in B_{c_{0}}(M, f)$ joining $p$ and $q$ for some constant $c_{0}>0$.

For the sake of simplicity, let $X=B_{c_{0}}(M, f)$ with the induced metric from the product $N \times M \times \cdots \times M$ and let $g=\left.E\right|_{B_{c_{0}}(M, f)}$. Note that $f^{-1}(\Delta)$ may be naturally identified with $g^{-1}(0)$. We claim that there is a sequence of connected paths $\bar{\gamma}_{k}:[0,1] \rightarrow g^{-1}[0,1 / k](k \geq 1)$ in $X$ such that $\bar{\gamma}_{k}(0)=p$ and $\bar{\gamma}_{k}(1)=q$ in the homotopy class of $\left[\bar{\gamma}_{0}\right]$ (keeping ends fixed).

We now prove (1.2.2) assuming this claim.

Note that the distance $d_{X}(A, B)>0$. Consider the function $\mu: X \rightarrow \mathbb{R}$ defined by $\mu(x)=d_{X}(x, A)-d_{X}(x, B)$. Note that the restriction of $\mu$ on $\bar{\gamma}_{k}$ satisfies that $\mu(p)<0$ and $\mu(q)>0$. Therefore, there is a point $x_{k} \in \bar{\gamma}_{k}$ so that $\mu\left(x_{k}\right)=0$. By passing to a subsequence, we may assume that $x_{k}$ converges to a point $x$. Note that $g\left(x_{k}\right)$ converges to zero. Thus, we have $x \in$ $g^{-1}(0)$ and $\mu(x)=0$. This is clearly a contradiction, since $A \cap B$ is empty.

By [25], Corollary 6.8 and the assumption $\lambda_{0} \geq 2$, we may choose a Morse function $h$ on $X$ such that $|g-h|<\frac{1}{10 k}$ on the compact sublevel set $X \leq c_{0}+\frac{1}{2}=\left\{x \in X: h(x) \leq c_{0}+\frac{1}{2}\right\}$ so that all critical points of $h$ in the set $h^{-1}\left(\left[\frac{1}{2 k}, c_{0}+\frac{1}{2}\right]\right)$ have Morse indices greater than 1. By Morse theory, we know that $h^{-1}\left(-\infty, c_{0}+\frac{1}{2}\right]$ is homotopy equivalent to $h^{-1}\left(-\infty, \frac{1}{2 k}\right]$ by gluing cells of dimensions at least 2 . Therefore, the relative homotopy group $\pi_{1}\left(h^{-1}\left(-\infty, c_{0}+\frac{1}{2}\right], h^{-1}\left(-\infty, \frac{1}{2 k}\right]\right)=0$. This implies that $\bar{\gamma}_{0}$ is homotopic to a path $\bar{\gamma}_{k}$ in $h^{-1}\left(-\infty, \frac{1}{2 k}\right]$ with the same end points $p, q$ fixed. This proves (1.2.2). 
(1.2.3) By Morse theory (cf. [25, 29]), it suffices to show that $E^{-1}(0)=$ $f^{-1}(\Delta) \subset B_{c}(M, f)$ has an open neighborhood $U \subset B_{c}(M, f)$ that deformation retracts to $f^{-1}(\Delta)$. Observe that, under the identification of $B_{c}(M, f)$ with $N \times M \times \cdots \times M$ with $k$ even, $f^{-1}(\Delta)$ is identified with a subset $S=\{(x, f(x), \cdots, f(x)) \in N \times(M \times M) \times \cdots \times(M \times M) \mid x \in$ $\left.f^{-1}(\Delta)\right\}$. So, the existence of such a neighborhood $U$ follows from Proposition 1.2 below.

(1.2.4) Note that $\pi_{i}(\Omega M) \cong \pi_{i+1}(M)$. Since $\Omega(M) \rightarrow P(M, f) \rightarrow N$ is the pullback of $\Omega(M) \rightarrow P(M) \rightarrow M \times M$ via $f: N \rightarrow M \times M$, there is a commutative diagram of homotopy exact sequences

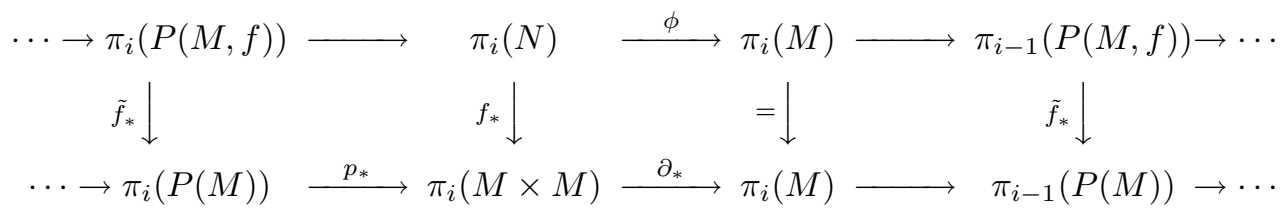

Since $P(M)$ is homotopic to $M$, it is easy to see that $p: P(M) \rightarrow M \times M$ is homotopic to the diagonal map. If $p_{i}$ is the projection of $M \times M$ to the $i$-th factor, then $\partial_{*}=\left(p_{1}\right)_{*}-\left(p_{2}\right)_{*}$. By the above diagram, we get the homomorphism $\phi=\left(p_{1} f\right)_{*}-\left(p_{2} f\right)_{*}$ (compare to [15]).

Let $X$ be a complete manifold, and let $f: N \rightarrow X \times X$ be an isometric immersion, where $N$ is a closed manifold. Consider the subset

$$
S=\left\{(x, f(x), \cdots, f(x)) \in N \times(X \times X) \times \cdots \times(X \times X) \mid x \in f^{-1}(\Delta)\right\} .
$$

Proposition 1.2. Let $X$ be a complete manifold, and let $f: N \rightarrow X \times X$ be an isometric immersion as above. Then, the subset $S$ in $N \times(X \times X) \times$ $\cdots \times(X \times X)$ is a deformation retraction of an open neighborhood $U$ if one of following conditions holds:

(1.3.1) $f$ is minimal;

(1.3.2) $N=N_{1} \times N_{1}$ and $f=f_{1} \times f_{1}$, where $f_{1}$ is an embedding;

(1.3.3) $f$ is totally geodesic.

Proof. If $f$ is minimal, then $f$ is harmonic and therefore, real analytic with respect to any harmonic coordinates in $N$ and $X \times X$ (cf. [21]). Therefore, the set $S$ considered is a real analytic variety. By [23], any compact real 
analytic variety is triangulable, as a subcomplex of the real analytic manifold $N \times(X \times X) \times \cdots \times(X \times X)$. Therefore, an open regular neighborhood of this subcomplex is a desired open neighborhood $U$.

In cases (1.3.2) and (1.3.3), we claim that $S$ is a closed manifold. We then take $U$, a smooth open tubular neighborhood of $S$, in $N \times(X \times X) \cdots \times$ $(X \times X)$. In case (1.3.2), $S$ is clearly diffeomorphic to $N$. Note that (1.3.3) is a special case of (1.3.1), but we prefer to give a more geometric proof. First, note that $S$ is diffeomorphic to $f^{-1}(\Delta)$. For any smooth open disk $D \subset N$ such that $f(D)$ is embedded in $X \times X$, the intersection $f(D) \cap \Delta$ is a totally geodesic submanifold, since locally it is given by the image $\exp _{p}\left(T_{p} f(D) \cap\right.$ $T_{p} \Delta$ ), where $\exp _{p}$ is the exponential map at $p \in f(D) \cap \Delta$, and $T_{p} f(D)$, $T_{p} \Delta$ the tangent spaces at $p$. Observe that $f: f^{-1}(\Delta) \cap D \rightarrow \Delta \cap f(D)$ is a diffeomorphism, proving that $f^{-1}(\Delta)$ is a manifold.

\section{Positive Curvature and Indices Estimates.}

As in Section 1, consider the energy function $E$ on $P(M, f)$. Note that the tangent space of $P(M ; f)$ at point $(x, \gamma)$ consists of $(v, W)$ where $v \in$ $T_{x} N$ and $W$ is a piecewise smooth vector field along $\gamma$ such that $f_{*}(v)=$ $(W(0), W(1))$. Since $f$ is an immersion, the tangent space can be identified as the space of vectors $(W(0), W(1))$. If $W$ is a parallel vector field along $\gamma$, then by the standard second variation formula, it follows that the Hessian of $E$ satisfies:

$$
E_{* *}(W, W)=\int_{0}^{1}-\langle R(\dot{\gamma}, W) W, \dot{\gamma}\rangle d t+\left\langle\alpha\left(f_{*}(v), f_{*}(v)\right),(-\dot{\gamma}(0), \dot{\gamma}(1))\right\rangle,
$$

where $\alpha(-,-)$ is the second fundamental form of $f$.

By definition, the index of $E$ at $(x, \gamma)$ is the maximal dimension of a subspace on which $E_{* *}$ is negative definite. Recall that the asymptotic index $\nu_{f}(x)$ is the maximal dimension of a subspace of $T_{x} N$ on which the second fundamental form $\alpha$ vanishes.

Lemma 2.1. Let $M$ be a complete $m$-manifold of positive $k$-th Ricci curvature, and let $f: N \rightarrow M \times M$ be a complete immersion with asymptotic in$\operatorname{dex} \nu_{f}$. Let $(x, \gamma)$ be any non-trivial critical point of $E$, with Morse index $I_{\gamma}$.

$$
I_{\gamma} \geq \nu_{f}-m-k+1
$$

(2.1.2) If $f=\left(f_{1}, f_{2}\right): N=N_{1} \times N_{2} \rightarrow M \times M$ such that each $f_{j}: N_{j} \rightarrow M$ is an immersion, then $I_{\gamma} \geq \nu_{f}-m-k+2$. 
Proof. (2.1.1) Let $V_{1}$ denote the set of parallel vector fields along and orthogonal to $\gamma$. Then, $\operatorname{dim} V_{1}=m-1$. Clearly, $V_{1}$ can be identified with $\left\{(v(0), v(1)), v \in V_{1}\right\}$. We now consider a symmetric quadratic form on $V_{1}$ defined by

$$
\langle A(v), v\rangle=\int_{0}^{1}-\langle R(\dot{\gamma}, W) W, \dot{\gamma}\rangle d t
$$

where $v=(W(0), W(1))$ and $\langle\cdot, \cdot\rangle$ is the metric induced from $T_{f(x)}(M \times M)$.

Let $V$ be some linear subspace of $V_{1}$ with maximal dimension such that the quadratic form is negative definite on $V$. Regard $V$ as a subspace of $T_{f(x)}(M \times M)$ with $f(x)=(\gamma(0), \gamma(1))$. Let $\mathcal{N}_{x} \subset T_{x} N$ denote the maximal subspace of $T_{x} N$ such that the restriction of the second fundamental form $\left.\alpha\right|_{\mathcal{N}_{x}}=0$. Since both $V$ and $f_{*}\left(\mathcal{N}_{x}\right)$ are normal to $(\dot{\gamma}(0),-\dot{\gamma}(1))$, the intersection $f_{*}\left(\mathcal{N}_{x}\right) \cap V$ has dimension

$$
\operatorname{dim}\left(f_{*}\left(\mathcal{N}_{x}\right) \cap V\right) \geq \nu_{f}+\operatorname{dim} V-2 m+1
$$

Note that,

$$
E_{* *}(W, W)=\langle A(v), v\rangle<0
$$

for any non-zero $v \in f_{*}\left(\mathcal{N}_{x}\right) \cap V$. We complete the proof by showing that $\operatorname{dim} V \geq m-k$.

Take an orthonormal basis for $V_{1}$ which are eigen vectors of $A$, $v_{1}, \ldots, v_{m-1}$. By the curvature assumption,

$$
\begin{aligned}
\sum_{i=1}^{k}\left\langle A\left(v_{i}\right), v_{i}\right\rangle & =\sum_{i=1}^{k} \int_{0}^{1}\left[-\left\langle R\left(\dot{\gamma}, v_{i}\right) v_{i}, \dot{\gamma}\right\rangle\right] d t \\
& =-\int_{0}^{1}\left(\sum_{i=1}^{k}\left\langle R\left(\dot{\gamma}, v_{i}\right) v_{i}, \dot{\gamma}\right\rangle\right) d t<0 .
\end{aligned}
$$

This implies that for some $i,\left\langle A\left(v_{i}\right), v_{i}\right\rangle<0$. Without loss of generality, we can assume $i=1$. Similarly, from

$$
\sum_{i=2}^{k+1}\left\langle A\left(v_{i}\right), v_{i}\right\rangle=-\int_{0}^{1}\left(\sum_{k=2}^{k+1}\left\langle R\left(\dot{\gamma}, v_{i}\right) v_{i}, \dot{\gamma}\right\rangle\right) d t<0
$$

we pick up, say $v_{2}$, such that $\left\langle A\left(v_{2}\right), v_{2}\right\rangle<0$. We repeat this operation until we pick up (after a suitable rearrangement of subindices) $v_{1}, \ldots, v_{m-k}$, with $\left\langle A\left(v_{i}\right), v_{i}\right\rangle<0,1 \leq i \leq m-k$. Then, $\langle A(v), v\rangle<0$ for all $v$ in the subspace spanned by $v_{1}, \ldots, v_{m-k}$ and thus, the desired result follows. 
(2.1.2) Note that $\gamma$ is a geodesic in $M$ such that $\dot{\gamma}(0) \perp\left(f_{1}\right)_{*}\left(T_{x_{1}} N_{1}\right)$ and $\dot{\gamma}(1) \perp\left(f_{2}\right)_{*}\left(T_{x_{2}} N_{2}\right)$, where $x=\left(x_{1}, x_{2}\right)$. Since both $(\dot{\gamma}(0), 0)$ and $(0, \dot{\gamma}(1))$ are normal to $f_{*}\left(T_{x} N\right)$ and $V$, the intersection $f_{*}\left(T_{x} N\right) \cap V$ has dimension,

$$
\operatorname{dim}\left(f_{*}\left(T_{x} N\right) \cap V\right) \geq \nu_{f}-m-k+2
$$

and thus, we obtain the desired result.

Remark 2.2. Let $f_{1}: N_{1} \rightarrow M$ be a minimal immersed hypersurface. Assume that $M$ has positive Ricci curvature. Let $f=\left(f_{1}, f_{1}\right): N=N_{1} \times$ $N_{1} \rightarrow M \times M$. Consider a non-trivial critical point for the energy function $(x, y, \gamma)$, with $x, y \in N_{1}$, and $\gamma$ being a geodesic joining $x$ and $y$, with $\dot{\gamma}(0) \perp f_{1 *}\left(T_{x} N_{1}\right)$ and $\dot{\gamma}(1) \perp f_{1 *}\left(T_{y} N_{1}\right)$. Given a parallel orthonormal frame $v_{1}, \cdots, v_{n_{1}-1}$ along $\gamma$ with $v_{i} \perp \dot{\gamma}$, the sum of the second variation formulas gives us ( $\alpha$ is the second fundamental form)

$$
\begin{aligned}
\sum_{i} E_{* *}\left(v_{i}, v_{i}\right) & =-\int_{0}^{1} \operatorname{Ric}(\dot{\gamma}) d t+\left\langle\sum_{i} \alpha\left(v_{i}, v_{i}\right), \dot{\gamma}\right\rangle(1)-\left\langle\sum_{i} \alpha\left(v_{i}, v_{i}\right), \dot{\gamma}\right\rangle(0) \\
& =-\int_{0}^{1} \operatorname{Ric}(\dot{\gamma}) d t<0 .
\end{aligned}
$$

Since $N_{1}$ is a hypersurface, $v_{i}(1)$ and $v_{i}(0)$ are tangent to $N_{1}$ for all $i$. Like above, we find that in this case, that the index of $\gamma$ is at least one.

\section{Proofs of Theorems A, B, C.}

Proof of Theorem A. (A1)-(A3) follow from (1.2.1)-(1.2.4), (2.1.1) for $k=1$.

Proof of Theorem B. (B1)-(B3) follows from (1.2.1)-(1.2.4), (2.1.2) for $k=1$.

One may derive (B4) from (B3). Here, we will present a direct approach.

Let $p_{1}: P(M, f) \rightarrow N_{1} \times N_{2} \rightarrow N_{1}$ denote the composition of the two projections. Note that $p_{1}$ is a fibration with fiber $V$, the pullback fibration 
fitting into the commutative diagram below

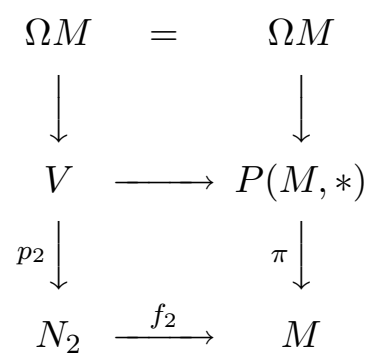

where the right-side is the principal path fibration. Since $P(M, *)$ is contractible, from the above diagram $V$ is the homotopy fiber of the map $f_{2}: N_{2} \rightarrow M$. By the fibration homotopy exact sequence, it follows that

$$
\pi_{i}(V) \approx \pi_{i+1}\left(M, N_{2}\right)
$$

for all $i$. This, together with the homotopy exact sequence for the fibration $V \rightarrow P(M, f) \stackrel{p_{1}}{\rightarrow} N_{1}$ and the long exact sequence for the map $i_{1}: f^{-1}(\Delta) \rightarrow$ $N_{1}$ give the commutative diagram:

$$
\begin{aligned}
& \pi_{i+1}\left(N_{1}\right) \rightarrow \pi_{i+1}\left(N_{1}, f^{-1}(\Delta)\right) \rightarrow \pi_{i}\left(f^{-1}(\Delta)\right) \rightarrow \pi_{i}\left(N_{1}\right) \rightarrow \pi_{i}\left(N_{1}, f^{-1}(\Delta)\right) \\
& =\downarrow \quad\left(f_{1}\right)_{*} \downarrow \quad \operatorname{surj} \downarrow \quad=\downarrow \quad\left(f_{1}\right)_{*} \downarrow \\
& \pi_{i+1}\left(N_{1}\right) \rightarrow \quad \pi_{i+1}\left(M, N_{2}\right) \quad \rightarrow \pi_{i}(P(M, f)) \rightarrow \pi_{i}\left(N_{1}\right) \rightarrow \quad \pi_{i}\left(M, N_{2}\right)
\end{aligned}
$$

The middle homomorphism is surjective for $i \leq \nu_{f}-m$, since by Theorem 1.1 and Lemma 2.1, we obtain $\pi_{i}\left(P(M, f), f^{-1}(\Delta)\right)=0$. From the 5-lemma and the commutative diagram above, we have

$$
\left(f_{1}\right)_{*}: \pi_{i}\left(N_{1}, f^{-1}(\Delta)\right) \rightarrow \pi_{i}\left(M, N_{2}\right)
$$

is an isomorphism for all $i \leq \nu_{f}-m$ and a surjection for $i=\nu_{f}-m+1$. This proves the desired result.

Proof of Theorem $\mathrm{C}$.

(C1)-(C3) follow from Theorem 1.1 and Lemma 2.1, and (C4) follows from the proof of (B4). 


\section{Proofs of Theorems $0.1-0.9$.}

Proof of Theorem 0.5.

Since $f: N \rightarrow M$ is an immersion, we will show that $f$ is a one-to-one map. Note that $f^{-1}(\Delta)=\{(x, x), x \in N\} \cup\{(x, y): f(x)=f(y), x \neq y\}$. Hence, if $f$ is not injective, then $f^{-1}(\Delta)$ is not connected; a contradiction to $(\mathrm{B} 2)$.

Proof of Theorem 0.6.

Consider the immersion $\left(f_{1}, f_{2}\right): N_{1} \times N_{2} \rightarrow M \times M$. By (B2), $\left(f_{1}, f_{2}\right)^{-1}(\Delta)$ is connected and thus $f_{1}^{-1}\left(f_{2}\left(N_{2}\right)\right)=p_{1}\left(\left(f_{1}, f_{2}\right)^{-1}(\Delta)\right)$ and $f_{2}^{-1}\left(f_{1}\left(N_{1}\right)\right)=p_{2}\left(\left(f_{1}, f_{2}\right)^{-1}(\Delta)\right)$ are connected.

Proof of Theorem 0.7 .

In (B4), consider $f_{1}=i_{1}: N_{1} \subset M$ and $f_{2}=i_{2}: N_{2} \subset M$. It is clear that $f^{-1}(\Delta)=N_{1} \cap N_{2}$ and then, the desired result follows.

Proof of Theorem 0.8 .

If $N_{2}$ is minimal, then we apply Theorem 0.7 to $N_{2}, N_{2}$. Note that $\pi_{i}\left(N_{2}, N_{2}\right)=0$ for all $i$.

By Theorem 0.7 and (0.8.1), we arrive at (0.8.2).

Proof of Theorem 0.1 .

Since Theorem 0.1 is trivial for $2 \nu_{f}-m+1=0$ and 1 , we may assume that $2 \nu_{f}-m+1>1$ i.e. $2 \nu_{f}>m$. By Theorem 0.5, $f$ is an embedding, allowing us to apply Theorem 0.8 to complete the proof.

The following is a generalization of Theorem 0.1, where the assumption on the simply connectedness of $M^{m}$ is dropped.

Theorem 4.1. Let $M^{m}$ be a closed manifold of positive sectional curvature, and let $f: N^{n} \rightarrow M^{m}$ be an isometric immersion of a closed manifold with asymptotic index $\nu_{f}$.

(4.1.1) $f_{*}: \pi_{q}\left(N^{n}\right) \rightarrow \pi_{q}\left(M^{m}\right)$ is an isomorphism (resp. a surjection) for 


$$
q=0,2, \ldots,\left(2 \nu_{f}-m\right)\left(\operatorname{resp} .2 \nu_{f}-m+1\right) .
$$

(4.1.2) If $2 \nu_{f}>m$, then $f_{*}: \pi_{1}\left(N^{n}\right) \rightarrow \pi_{1}\left(M^{m}\right)$ is an injection.

Proof. (4.1.1) Let $\pi_{M}: \tilde{M}^{m} \rightarrow M^{m}$ denote the Riemannian universal covering map, and let $\hat{\pi}: \hat{N}^{n} \rightarrow N$ denote the pullback covering map by $f$. Then, $\hat{N}^{n}$ is a finite covering space over $N^{n}$. Consider the isometric immersion: $\hat{f}: \hat{N}^{n} \rightarrow \tilde{M}^{m}$, where $\hat{f}: \hat{N}^{n} \rightarrow \tilde{M}^{m}$ be lifting of $f: N^{n} \rightarrow M^{m}$. Since $\tilde{N}^{n}$ is closed, we may apply Theorem 0.1 to conclude that $\hat{f}$ is $\left(2 \nu_{f}-m+1\right)$-connected. The proof is then complete by observing that $(\hat{\pi})_{*}: \pi_{q}\left(\hat{N}^{n}\right) \rightarrow \pi_{q}\left(N^{n}\right)\left(\operatorname{resp} .\left(\pi_{M}\right)_{*}: \pi_{q}\left(\tilde{M}^{m}\right) \rightarrow \pi_{q}\left(M^{m}\right)\right)$ are isomorphisms for $q \neq 1$.

(4.1.2) By Theorem 0.5, $\hat{f}: \hat{N}^{n} \rightarrow \tilde{M}^{m}$ is an embedding. Hence, we may apply Theorem 0.8 to conclude that $\hat{N}^{n}$ is simply connected and thus the desired result.

In the proof of Theorem 0.2, we use the following theorem ([8] Prop. 6, compare [5]):

Theorem $4.2([8])$. Let $f: N^{n} \rightarrow M^{n+p}$ be an isometric immersion of a closed manifold with non-positive extrinsic curvature. Then, the asymptotic index $\nu_{f} \geq n-p$.

Proof of Theorem 0.2 .

By Theorem 4.2, the asymptotic index $\nu_{f} \geq n-p$. By Theorem 0.1, $f$ is $[2(n-p)-(n+p)+1]=(n-3 p+1)$-connected.

\section{Proof of Theorem 0.3 .}

We first consider the non-positive extrinsic curvature case. By Theorem $0.2, f$ is $(n-3 p+1)$-connected. Since $n \geq 7 p-2, n-3 p+1 \geq \frac{n+p}{2}$. By the Poincaré duality and Hurewicz theorem, it is easy to see that $N^{n}$ is a homotopy sphere if and only if $M^{n+p}$ is a homotopy sphere. The desired result follows since by Smale ([30]) a homotopy sphere of dimension at least 5 is homeomorphic to a sphere. 
Proof of Theorem 0.9 .

If $f^{-1}(\Delta)=\emptyset$, then $I(x, \gamma)=0$, where $(x, \gamma)$ is a critical point at which $E$ achieves the absolute minimal value. Let $V_{1}$ be as in the proof of (2.1.1). Then, the standard Synge type argument implies that $V_{1} \cap f_{*}\left(T_{x}(M)\right) \neq 0$, making $I(x, \gamma) \geq 1$; which is not possible.

Proof of Corollary 0.10.

Set $N_{1}=N_{2}=N$, and let $i: N \rightarrow M$ be the inclusion. Set $f=$ $i \times i: N \times N \rightarrow M \times M$. Since $f^{-1}(\Delta)=\Delta_{N}$ and $p_{1}: \Delta_{N} \rightarrow N$ is a diffeomorphism, we have $\pi_{1}\left(N, f^{-1}(\Delta)\right)=0$. By Remark 2.2, the index of each critical point is at least 1 . Thus, the same proof as Theorem B leads to the conclusion that

$$
\pi_{1}\left(N, f^{-1}(\Delta)\right) \rightarrow \pi_{1}(M, N)
$$

is surjective. This proves that $\pi_{1}(M, N)=0$. The desired result follows.

\section{Positively Curved Manifolds With Maximal $p$-Symmetry Rank.}

The symmetry rank of a Riemannian manifold $M$ is defined as the rank of a maximal torus of the isometry group $\operatorname{Isom}(M)$. For any prime $p$, the $p$-symmetry rank of $M$ is the maximal rank of elementary $p$-subgroups of $\operatorname{Isom}(M)$.

Recently, the classification of positively curved manifolds with large symmetry rank has received a lot of attention (cf. [16, 17, 19, 27, 7, 33]). It seems that in these works, the connectedness theorems for totally geodesic submanifolds plays an important role.

The classification of positively curved manifolds with large $p$-symmetry rank was considered in [6] and the connectedness theorems, among others, were applied to obtain the homeomorphic classification in even-dimensions for $p$ greater than a constant depending only on the dimension.

The purpose of this section is to partially extend, using the connectedness theorems, the above classification result of [6] to one that includes all primes.

Theorem 5.1 (Maximal $p$-rank). Let $M^{m}$ be a closed simply connected manifold of positive sectional curvature. Assume that $M^{m}$ admits an effective isometric $\mathbb{Z}_{p}^{k}$-action with a non-empty fixed point set.

(5.1.1) If $p=2$, then $k \leq m$ and "=" implies that $M^{m}$ is homeomorphic to a sphere. 
(5.1.2) If $p>2$, then $k \leq m / 2$ and ' $=$ ' implies that $M^{m}$ is homeomorphic to a sphere or a complex projective space.

Note that (5.1.1) provides a partial solution to a problem of Petersen's (p. 108, [26]): Is a topological $m$-sphere the only positively curved manifold which admits an isometric $\mathbb{Z}_{2}^{m}$-action?

Theorem 5.1 may apply in the following situations.

Theorem 5.2. Let $M^{2 n}$ be a closed simply connected manifold of positive sectional curvature. Assume that $M^{n}$ admits an effective isometric $\mathbb{Z}_{p}^{k}$ action. Then, the fixed point set is not empty under any of the following conditions:

(5.2.1) The Euler characteristic $\chi\left(M^{2 n}\right) \neq 0 \bmod (p)$.

(5.2.2) $p \geq p(n)$, where $p(n)$ is a constant depending only on $m$.

Note that (5.2.2) and (5.1.2) imply the maximal $p$-rank theorem of [6]. The proof of (5.1.1) requires the following.

Theorem 5.3 (Totally geodesic hypersurface rigidity). Let $M$ be a closed manifold of positive sectional curvature. If $M$ has a closed totally geodesic hypersurface, then either $M$ (resp. $N$ ) or its double covering is homeomorphic to a sphere simultaneously.

The natural isometric embedding of $\mathbb{R} P^{n} \hookrightarrow \mathbb{R} P^{n+1}$ shows that Theorem 5.3 is optimal. Note that Theorem 5.3 was obtained by [34] for the following case: $M$ is simply connected and $N$ is orientable. One can easily extend it to Theorem 5.3 via using Synge theorem and Theorem 0.8 .

Proof of Theorem 5.3. If $n$ is even, then $\operatorname{dim}(N)$ is odd and thus, $N$ is orientable (Synge theorem). By [34], we may assume that $M$ is not orientable, and thus $\pi_{1}(M) \cong \mathbb{Z}_{2}$. Let $\tilde{M}$ denote the double covering of $M$, and let $\tilde{N}$ denote the inverse image of $N$ in $\tilde{M}$. Because the inclusion $N \hookrightarrow M$ is at least 2-connected (Theorem 0.8), $\tilde{N} \rightarrow N$ is a double covering. By [34], we conclude that both $\tilde{M}$ and $\tilde{N}$ are homeomorphic to spheres.

If $n$ is odd, then $\tilde{M}$ is orientable. Because $\operatorname{dim}(N)$ is even, either $N$ is orientable and thus $M$ is simply connected (Theorem 0.8), or $\pi_{1}(N) \cong$ $\pi_{1}(M) \cong \mathbb{Z}_{2}$. As in the above, we conclude that a double covering of $M$ and a double covering space of $N$ are homeomorphic to spheres. 
Proof of Theorem 5.1.

Consider the orthogonal representation of $\mathbb{Z}_{p}^{k}$ in $O(m)$ at any fixed point. Then, $k \leq m$ for $p=2$ and $k \leq m / 2$ for $p>2$.

Case 1. Assume that $p=2$. From the orthogonal representation, it is easy to see that there is a $\mathbb{Z}_{2}$ subgroup whose fixed point component has codimension one. This implies a closed totally geodesic hypersurface. By now, the desired result follows from Theorem 5.3.

Case 2. If assuming Proposition 1.3 in [6], then the proof below is almost identical to that of the maximal $p$-rank Theorem in [6]. Hence, we will only give an outline of the proof; see [6] for details.

a. Assume that $m=2 n$. From the isotropy representation at a fixed point, we conclude that a sequence of closed totally geodesic submanifolds

$$
M^{4} \subset M^{6} \subset \cdots \subset M^{2 n}=M^{m}
$$

where $M^{2 i}$ is a fixed point component of a $\mathbb{Z}_{p}$-rank $(n-i)$ subgroup in $\mathbb{Z}_{p}^{n}$. By $(0.8 .1) M^{2 i} \rightarrow M^{2 i+2}$ is $(2 i-1)$-connected. Therefore, all $M^{2 i}(i \geq 2)$ are simply connected.

As shown in [6], $M^{m}$ is homeomorphic to a sphere or a complex projective space if $M^{4}$ is homeomorphic to $S^{4}$ or $\mathbb{C} P^{2}$; the proof of the later in [6] requires that $p \geq p(4)$. Since we allow all prime $p$ to exist, we give the following proof.

By the isotropy representation, it is easy to see that there is another

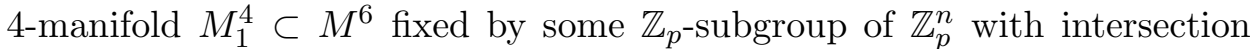
$M_{1}^{4} \cap M^{4}=M^{2}$. By (0.8.2), it follows that $i: M^{2}=M_{1}^{4} \cap M^{4} \rightarrow M^{4}$ is a 2-equivalence. Thus, $M^{2}$ is simply connected and so $M^{2}=S^{2}$. This implies that $M^{4}$ is simply connected and the second Betti number $b_{2}\left(M^{4}\right) \leq 1$. By [11], $M^{4}$ is either homeomorphic to $S^{4}$ or $\mathbb{C} P^{2}$.

b. Assume that $m=2 n+1$. From the isotropy representation at a fixed point, we conclude that a sequence of closed totally geodesic submanifolds

$$
M^{3} \subset M^{5} \subset \cdots \subset M^{2 n+1}=M^{m}
$$

where $M^{2 i+1}$ is a fixed point component of a $\mathbb{Z}_{p}$-rank $(n-i)$ subgroup in $\mathbb{Z}_{p}^{n}$. By (0.8.1), $M^{2 i-1} \rightarrow M^{2 i+1}$ is $(2 i-1)$-connected. Therefore, all $M^{2 i+1}$ $(i \geq 2)$ are simply connected. By Hamilton $([19]), M^{3}$ is homeomorphic to a sphere. Like the above, by $(0.8 .1)$, we then conclude that $M^{m}$ is homeomorphic to a sphere. 
Proof of Theorem 5.2.

Recall that the Euler characteristic $\chi(M)=\chi\left(M^{\mathbb{Z}_{p}^{k}}\right) \bmod (p)([4])$. This implies that the fixed point set $M^{\mathbb{Z}_{p}^{k}} \neq \emptyset$. The proof of (5.2.2) may be found in $[6]$.

\section{Acknowledgment.}

The authors would like to thank Jeff Cheeger, Shingshen Chern, William Fulton, Karsten Grove, Gudlaugur Thorbergsson, Burhard Wilking for helpful comments and remarks. The authors would like to particularly thank Karsten Grove for many constructive suggestions on this revision. The authors would like to thank Detang Zhou for some helpful discussion and bringing [29] to our attention and Jon Wolfson for some helpful discussions concerning [29]. The first author would like to thank Luis Florit for useful discussions on isometric immersions, and the third author would like to thank Burkhard Wilking for informing him of main results in [33].

\section{References.}

[1] D. Bao; S. S. Chern; Z. Shen, An introduction to Riemann-Finsler geometry, Grad. Texts in Math. 200 (2000), Springer-Verlag.

[2] W. Barth, Transplating cohomology classes in complex projective space, Amer. J. Math. 92 (1970), 951-967.

[3] R. Bott, On a theorem of Lefschetz, Mich. Math. J. 6 (1959), 211-216.

[4] G. Bredon, Introduction to compact transformation groups, Academic Press 48 (1972).

[5] S.S. Chern; N. H. Kuiper, Some theorems on the isometric embedding of compact Riemannian manifolds in Euclidean space, Ann. of Math $\mathbf{5 6}$ (1952), 422-430.

[6] F. Fang; X. Rong, Positively curved manifolds of maximal discrete symmetry rank, American J. Math. 126 (2004), 227-245.

[7] F. Fang; X. Rong, Homeomorphism classification of positively curved manifolds of almost maximal symmetry rank, Math. Ann. 332 (1) (2005), 81-101.

[8] L. Florit, On submanifolds with nonpositive extrinsic curvature, Math. Ann. 298 (1994), 187-192. 
[9] T. Frankel, Manifolds of positive curvature, Pacific J. Math. 11 (1961), $165-174$.

[10] T. Frankel, On the fundamental groups of a compact minimal submanifold, Ann. Math. 83 (1966), 68-73.

[11] M. Freedman, Topology of Four Manifolds, J. Diff. Geom. 28 (1982), $357-453$.

[12] W. Fulton, On the topology of algebraic varieties, Proc. Symp. in Pure Math. 46 (1987), 15-46.

[13] W. Fulton; J. Hansen, A connectedness theorem for projective varieties, with applications to intersections and singularities of mappings, Ann. of Math. 110 (1979), 159-166.

[14] W. Fulton; R. Lazarsfeld, Connectivity and Its Applications in Algebraic Geometry, Lecture Notes in Mathematics 862, Springer-Verlag, 26-92.

[15] K. Grove, Geodesics satisfying general boundary conditions, Comment. Math. Helv. (1973), 376-381.

[16] K. Grove, Geometry of, and via symmetries, Univ. Lecture Ser., Amer. Math. Soc., Providence, RT. 27 (2002), 31-53.

[17] K. Grove; C. Searle, Positively curved manifolds with maximal symmetry-rank, J. Pure Appl. Alg. 91 (1994), 137-142.

[18] M. Goresky; R. MacPherson, Stratified Morse theory, Springer-Verlag, Berlin Heidelberg 1988.

[19] R. Hamilton, Three-manifolds with positive Ricci curvature, J. Diff. Geom. 17 (1982), 255-306.

[20] W. Hsiang; B. Kleiner, On the topology of positively curved 4-manifolds with symmetry, J. Diff. Geom. 30 (1989), 615-621.

[21] J. Jost, Riemannian geometry and geometric analysis, Second Edition, Universitext, Springer-Verlag, Berlin (1998).

[22] S. Lefschetz, L'analysis situs et la geometrie algebrique, GauthierVillars, Paris (1924).

[23] S. Lojasiewicz, Triangulation of semi-analytic sets, Ann. Scu. Norm. di Pisa (1965), 449- 474. 
[24] M. Morse, A fundamental class of geodesics on any closed surfaces of genus greater than one, Trans. Amer. Math. Soc. 26 (1924), 25-60.

[25] J. Milnor, Morse theory, Ann. Math. Stud. Princeton University Press (1963).

[26] P. Petersen, Comparison geometry problem list, Riemannian Geometry (Waterloo ON, 1993), Fields Inst. Monogr. 4 (AMS 1996), 87-115.

[27] P. Petersen; F. Wilhelm, On Frankel's Theorem, Canad. Math. Bulletin 46 (2003), 130-139.

[28] X. Rong, Positively curved manifolds with almost maximal symmetry rank, Geometriae Dedicata 59 (2002), 157-182.

[29] R. Schoen; J. Wolfson, Theorems of Barth-Lefschetz types and Morse theory on the spaces of paths, Math. Zeit. 229 (1998), 77-89.

[30] S. Smale, Generalized Poincaré conjecture in dimension > 4, Ann. of Math. 74 (1961), 391-466.

[31] Z. Shen, On complete manifolds of nonnegative kth-Ricci curvature, Trans of A.M.S. 338 (1993), 289-310.

[32] J. Synge, On the connectivity of spaces of positive curvature, Quarterly J. Math. Oxford 7 (1936), 316-320.

[33] B. Wilking, Torus actions on manifolds of positive sectional curvature, Acta Math. 191 (2003), 259-297.

[34] H. Wu, Manifolds of partially positive curvature, Indiana Univ. Math. J 36 (1987), 525-548.

Nankai Institute of Mathematics

NANKAI UNIVERSITY

TianJin 300071, P.R.C.

E-mail address: ffang@nankai.edu.cn

Instituto DE MATEMÁtica

Universidade Federal Fluminense

Niterói, RJ

BRASIL

E-mail address: fuquan_fang@hotmail.com 
Departamento de Análise

Universidade Federal Fluminense (UFF),

Niterói, 24020

RJ BRAZIL

E-mail address: s_mendonca@hotmail.com; mendonca@mat.uff.br

Mathematics Department

Capital Normal University

BeiJing, P.R.C.

Mathematics Department

Rutgers UNIVERSITY

New Brunswick, NJ 08903, U.S.A

E-mail address: rong@math.rutgers.edu

Received August 14, 2003. 
